\title{
Application of Algorithm Target Change of the Filled Cells of the Matrix for a One-Dimensional Bending Element in the Problems Of Structural Mechanics
}

\author{
Katerinina S.Yu. \\ Volgograd State Technical University \\ Volgograd, Russian Federation \\ kat_svetlana@mail.ru \\ Rekunov S.S. \\ Volgograd State Technical University \\ Volgograd, Russian Federation \\ rekunoff@mail.ru \\ Konovalov O.V. \\ Volgograd State Technical University \\ Volgograd, Russian Federation \\ kov373@mail.ru
}

\author{
Voronkova G.V \\ Volgograd State Technical University \\ Volgograd, Russian Federation \\ vvgala@mail.ru \\ Arzamaskova L.M. \\ Volgograd State Technical University \\ Volgograd, Russian Federation \\ lar5832@yandex.ru \\ Evdokimov E.E. \\ Volgograd State Technical University \\ Volgograd, Russian Federation \\ smat_b101@rambler.ru
}

\begin{abstract}
The technology of design is considered on a basic resolving matrix for calculations of rod systems with an application of an algorithm of target change of matrix cells. This technology allows creating automated systems for solving a wide range of engineering tasks, including the tasks of building mechanics.
\end{abstract}

Keywords - resolving matrix, border conditions, computer modelling, one-dimensional element component

\section{INTRODUCTION}

Automation of calculations of building structures taking into account the use of modern information technologies is an urgent task.

Rapid dissemination of the latest software for system-wide and applied applications in the information services market and their numerous subsequent modifications in accordance with the requirements of the consumer, provide researchers with ample opportunities to use non-traditional approaches to solve it. Existing automated calculation systems, developed on the basis of traditional technologies, usually use computers as high-speed computing mechanisms and not as a powerful tool for computer modeling of various processes, including the process of studying the behavior of structures under various effects. The inefficiency of such systems is mainly determined by the need to input a considerable amount of input data from the keyboard, especially with their variations.
With a proper choice of the tool and effective use of the entire arsenal of unique opportunities provided to the developer by the medium of this tool, you can enter a new level of computerization of research activities.

It should be taken into account that the methodology for performing a calculation chosen for a specific task is determined both by the initial conditions of the task, and by the type of software and the capabilities of the computer equipment. For example, the latest versions of the table processor Excel [1, 2, 7] give an opportunity to enter a fundamentally new level of automation of volumetric systems of calculation of almost any direction and specialization. This allows us to organize a full-fledged process of investigating the reaction of the elements of the rod structures to the entire spectrum of possible impacts $[4,5,6,8]$ on the basis of a standard personal computer.

\section{METHODS AND MATERIALS}

When developing any specialized system of automation of calculations, the developers face the tasks of a general nature:

- organization of a "friendly" dialogue with the user when entering the source information;

- formation of source data sets;

- formation of the resolving matrix and the vector of the right-hand sides of systems 
- algebraic equations;

- system decision;

- development of the "quick response" algorithm for changing the values of the initial data and its implementation;

- formation of a block of calculation results, preferably with their visualization and automation of the procedure for estimating the accuracy of the results obtained.

The ultimate goal of this stage of research is the development of a universal technology of engineering calculations in a widespread MS Office Excel environment that allows creating automated systems for solving a wide range of engineering tasks, including the tasks of construction mechanics.

An algorithm for achieving the goal includes:

- automation of the process of input of initial data using visual control over the reliability of the input information;

- automation of the process of filling the elements of matrices (or vectors);

- development of a rational technology for transforming matrices and performing the necessary matrix operations;

- development of a rational technology for recording changes in the boundary conditions;

- graphical interpretation of calculation results, which allows the user to monitor the behavior of the calculated structure in the course of dynamic changes in its geometric, physical and topological parameters, as well as parameters of external influences;

- automation of comparative estimates of the accuracy of calculations for the proposed and classical methods.

\section{RESULTS}

This approach is well applicable in the study of stressstrain state of regular and biregular rod systems. These systems are widely used in engineering practice because of their characteristics such as the ability to cover large spans, the use of line production, unification and reduction of installation time, reducing the total cost of construction. At the same time, when calculating such constructions by exact methods due to a large number of nodes and elements, technical and mathematical difficulties arise. The proposed technique avoids some of them.

Earlier, in works $[1,2]$ the basic technique of spline approximations for the calculation of two-dimensional structures was developed, which makes it possible to implement at the program level an algorithm for constructing cubic splines with the aim of obtaining a discrete analogue of the basic differential equation of a one-dimensional bending element [2].

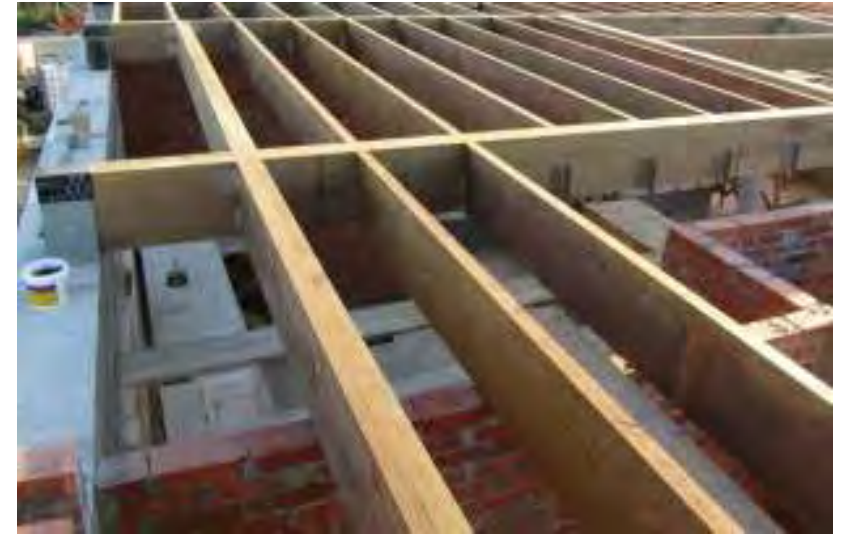

Fig.1. The core of the resolving matrix

Description of the technology of designing the basic resolving matrix for calculating the pivot systems in a table processor environment of MS Excel [3,9] will be expounded by the example of a single-span beam (i.e., a one-dimensional bending element, Fig. 2).

We put on the element's axis $n$ uneven segments (in this case, $n=10$ ) with length $h_{i}: h_{1}=0.1, h_{2}=0.05, h_{3}=0.11, h_{4}=0.09$, $h_{5}=0.08, h_{6}=0.12, h_{7}=0.15, h_{8}=0.09, h_{9}=0.11, h_{10}=0.1$.

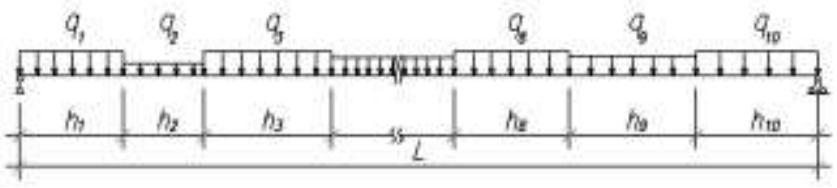

Fig. 2. Single-span beam

We denote the external load as $q_{i}$ and the ratio of the preceding length of the segment to the next one as $k_{i}=h_{\mathrm{i}} / h_{\mathrm{i}+1}$. The calculated values of these coefficients are included in the corresponding rows of the table (Fig. 3).

TABLE I. SOURCE DATA

\begin{tabular}{|l|l|l|l|l|l|l|l|l|l|}
\hline \multicolumn{10}{|c|}{ Load } \\
\hline$q_{1}$ & $q_{2}$ & $q_{3}$ & $q_{4}$ & $q_{5}$ & $q_{6}$ & $q_{7}$ & $q_{8}$ & $q_{9}$ & $q_{10}$ \\
\hline 1 & 1 & 1 & 1 & 1 & 1 & 1 & 1 & 1 & 1 \\
\hline \multicolumn{10}{|c|}{ Geometric parameter } \\
\hline$h_{1}$ & $h_{2}$ & $h_{3}$ & $h_{4}$ & $h_{5}$ & $h_{6}$ & $h_{7}$ & $h_{8}$ & $h_{9}$ & $h_{10}$ \\
\hline 0.10 & 0.05 & 0.09 & 0.12 & 0.09 & 0.10 & 0.10 & 0.11 & 0.08 & 0.15 \\
\hline \multicolumn{10}{|c|}{$\boldsymbol{k}_{\boldsymbol{i}}=\boldsymbol{h}_{\mathbf{i}} / \boldsymbol{h}_{\mathbf{i}+\mathbf{1}}$} \\
\hline 2.00 & 0.56 & 0.75 & 1.41 & 0.85 & 1.00 & 0.91 & 1.38 & 0.53 & 0.53 \\
\hline
\end{tabular}

To form the basic resolving matrix, the cells of the table are filled with the values of the coefficients for unknown system equations formed on the basis of discrete analogs of the differential equation $\alpha \omega^{\xi \xi}=-\theta$, describing deformed (deflections) and the stressed (moment) state of the bent element [1]:

$$
\begin{gathered}
y_{i-1}-y_{i-}-y_{i+}-y_{i+1}-h_{i+1}\left(\varphi_{i-} k_{i h}-\varphi_{i+}\right)= \\
=-\left(h^{2}{ }_{i+1} / 6\right)\left[k^{2}{ }_{i h}\left(2 m_{i-}+m_{i-1}\right)+\left(2 m_{i+}+m_{i+1}\right)\right] \\
m_{i-1}-m_{i-}-m_{i+}-m_{i+1}-h_{i+1}\left(Q_{i-} k_{i h}-Q_{i+}\right)=
\end{gathered}
$$


$=-\left(h^{2}{ }_{i+1} / 6\right)\left[k^{2}{ }_{i h}\left(2 q_{i-}+q_{i-1}\right)+\left(2 q_{i+}+q_{i+1}\right)\right]$.

For support nodes with hinge and uniformly distributed load $q$, believig that:

$$
\begin{aligned}
& y_{i-1}=y_{i-}=y_{i^{+}}=0 ; \\
& m_{i-1}-m_{i-}-m_{i+}=0 ; \\
& \varphi_{i^{-}}-\varphi_{i^{+}}=0 ; \\
& Q_{1-}=0 ; \\
& q_{i-1}-q_{i-}=0 ; \\
& q_{i+1}-q_{i+}=q,
\end{aligned}
$$

we get:

$$
\begin{aligned}
& i=0: y_{1}+h \varphi_{0}=-\left(h^{2} / 6\right) m_{1} ; \\
& i=10: y_{9}+h \varphi_{10}=-\left(h^{2} / 6\right) m_{10} ; \\
& i=0: m_{1}+h Q_{0}=-\left(h^{2} / 6\right) q_{1} ; \\
& i=10: m_{9}+h Q_{10}=-\left(h^{2} / 6\right) q_{10} .
\end{aligned}
$$

According to the generally accepted method of matrix formation, their elements - memory cells - are filled with the values of these coefficients as a result of the implementation of the program code of the corresponding standard programs $[1,4,10]$. And since the values of the coefficients change when any input parameter changes, then the variation of at least one parameter leads to the necessity of restarting in full the program code for forming matrices.

This significantly reduces the speed of execution of calculations. The solution to this problem is the creation of a special algorithm for the target change in the filled cells of the matrix, depending on the variable that is used to fill in the cells with the cell addresses, in which the current values of the variables are located.

Filling non-zero cells of the matrix with reference formulas is a long and painstaking task. So using the ability of spreadsheets to copy a cell with a link formula, automatically changing the addresses of cells included in the formula, causes the developer to be very responsible when constructing such formula. The Excel spreadsheet processor environment allows one to abandon the generally accepted technique of forming the resolving matrix. The universalization of such a technology that realizes discrete analogs in the form of (1) and (2) is that for each type of load and a specific boundary condition, a separate resolving matrix is created, resulting from the summation of the two matrices:

- basic, or base matrix whose core is invariant for all other matrices and does not depend on boundary conditions, while the extreme columns and rows of this matrix are

\begin{tabular}{|c|c|c|c|c|c|c|c|c|c|c|c|c|c|c|c|c|c|c|c|c|c|c|}
\hline$x$ & 01 & & 0 & 9 & 0 & 9 & 0 & $\theta$ & O & 9 & 0 & & 902 & 10 & 0 & 0 & 9 & 0 & 0 & 0 & 0 & \\
\hline$r_{4}$ & D & 0 & 7 & 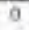 & 0 & 8 & 0 & 9 & 0 & 9 & 0 & 0 & 0 & 0 & 0 & 0 & 0 & 8 & 0 & 7 & & \\
\hline$\gamma_{1}$ & II & a & 0 & a & 0 & 0 & 6 & 0 & 0 & จ & 0 & 0 & & 0 & 0 & 0 & i) & 6 & 0 & 0 & D & \\
\hline$r_{1}$ & 0 & 0 & 0 & 0 & 0 & $\theta$ & 0 & 9 & 0 & 0 & 0 & 0 & & 0 & 0 & 0 & 0 & 0 & 0 & $\theta$ & e & \\
\hline$n$ & a & v & a & a & 0 & 0 & g & o & 0 & 0 & n & & & e & $a$ & D & D & a & a & 0 & & \\
\hline$\gamma_{1}$ & 0 & 0 & 0 & 0 & 0 & g & 0 & a & 0 & 9 & 0 & g & & 8 & 0 & 0 & 0 & g & 0 & ! & & \\
\hline f. & 0 & 0 & 0 & a & 0 & 0 & 0 & 0 & 0 & 0 & 0 & 0 & & 0 & 0 & 6 & 0 & 0 & 0 & 0 & & \\
\hline fi & v & 0 & o & a & 0 & 9 & 0 & q & n & 0 & 0 & ( & & 0 & 0 & 0 & 0 & 6 & 0 & & & \\
\hline & e & (1) & n & 0 & D & e & II & o & 0 & 0 & D & 0 & & a & a & D & g & e & 0 & D & & \\
\hline 6. & a & a & 0 & 오 & 0 & e & 0 & $\theta$ & 0 & 오 & 0 & a & 와 & 0 & 2 & 0 & 0 & 0 & 0 & 0 & 0 & \\
\hline क्या & 0 & 0 & $\theta$ & 0 & 0 & 0 & 0 & 0 & 0 & 1 & 0,1 & 0 & 9 & 0 & 0 & 0 & 0 & 0 & 0 & 0 & 6002 & \\
\hline Q & 0 & 0 & 0 & 9 & 0 & 0 & 0 & 0 & 0 & 9 & 0 & 01 & 10 & 0 & 0 & 6 & 0 & 0 & 0 & 0 & 0 & \\
\hline & 0 & ia & a & 0 & a & Q & 0 & 0 & o & 0 & 0 & 4 & 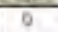 & 6 & 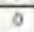 & 6 & 0 & 10 & $\bar{D}$ & 6 & 8 & \\
\hline$\pi$ & (i) & 0 & a & 0 & v & g & 0 & 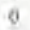 & 0 & 0 & 0 & c) & & & 0 & 0 & 0 & 0 & 0 & 0 & & \\
\hline & 0 & o & & 0 & & 0 & 0 & a & & 0 & & 0 & & & a & 0 & 0 & e & o & & & \\
\hline & 0 & 0 & 0 & 0 & i & 9 & 0 & 0 & 0 & 0 & 0 & i & & 0 & 0 & 0 & 0 & 0 & 0 & & & \\
\hline$m$ & a & 0 & 0 & 0 & 1 & 0 & 0 & 0 & 0 & 0 & 0 & 0 & & & 0 & D & 0 & 0 & D & is & 0 & \\
\hline 76 & 0 & 0 & & 0 & & 0 & 0 & 0 & & 0 & 0 & 0 & & & 0 & 0 & 0 & 0 & 0 & & & \\
\hline & $\theta$ & a & 0 & a & 1 & 0 & 0 & 0 & 0 & 0 & a & 0 & 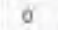 & e & a & e & a & e & a & & & \\
\hline$i n$, & 0 & 0 & 0 & อ & 0 & 0 & 0 & 8 & 10 & 9 & d & 0 & & a & a & 0 & 9 & 0 & 0 & & & \\
\hline & 0 & 0 & 1) & ฮ & 1) & a & 0 & a & 1 & 0 & 0 & 8 & & & 9 & 옥 & 0 & 6 & b & 으 & o & \\
\hline & i & 0 & 0 & 0 & 0 & 0 & 0 & 0 & & $\theta$ & & s & & $\theta$ & 0 & 0 & 9 & 0 & 0 & 0 & 10 & \\
\hline
\end{tabular}
zero (Fig. 3)

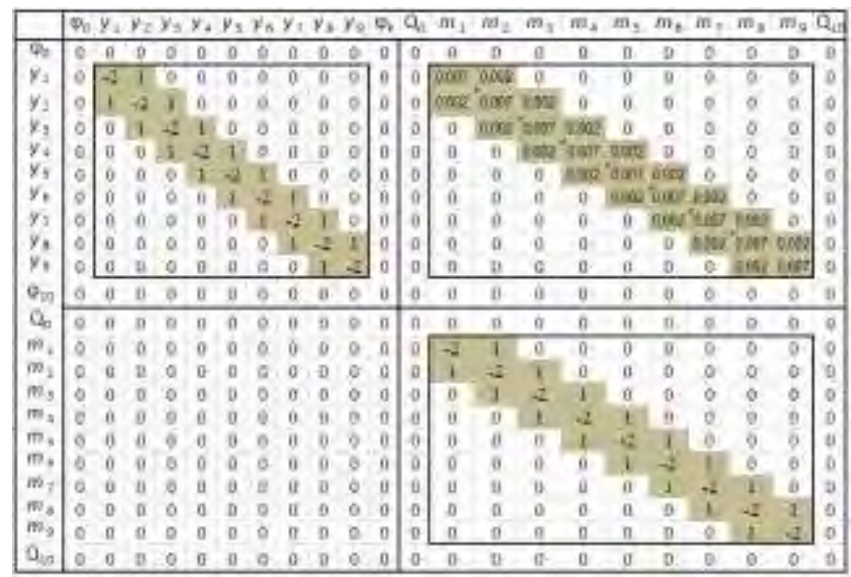

Fig.3. The core of the resolving matrix

- Fig. 4, the core of which is filled with zeros, and several cells of its extreme columns and rows are filled with such values that, as a result of summation of the given matrix with the base, yield the final matrix (Fig. 5), taking into account specific boundary conditions.

Fig.4. The additional matrix of the hinge

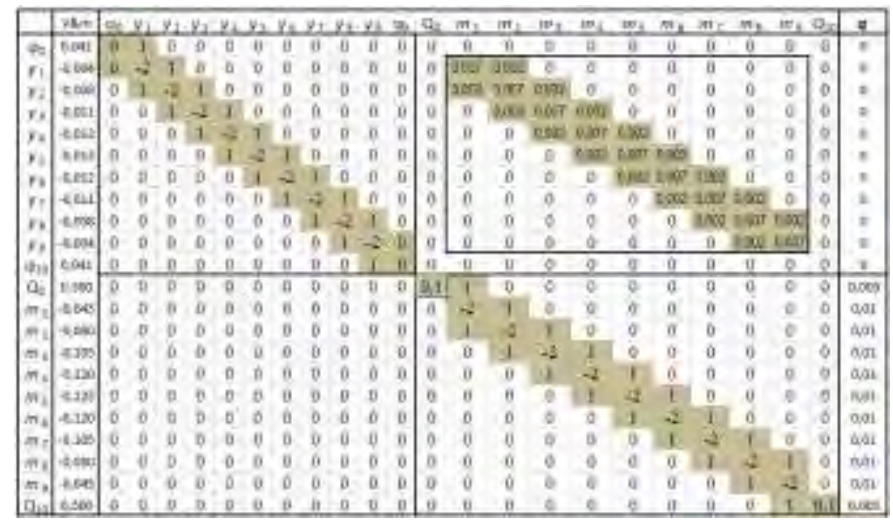

Fig.5. The final matrix of the hinged boundary conditions 
Next, the vector of the right-hand sides of the generalized equations (1), (2) is formed, i.e. the vector of external load q, which is shown on the right of the resolving matrix (Fig. 6). Then the inverse of the resolving matrix is calculated (Fig. 7).

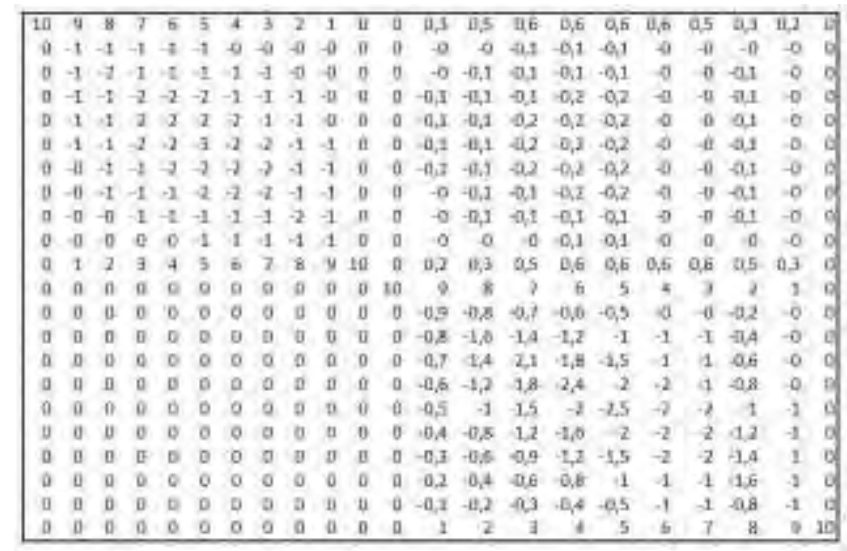

Fig. 6. The inverse of the resolving matrix

Finally, the result of multiplying an inverse matrix by vector q determines a vector whose elements are the values of unknown unknowns, i.e. $y_{1} \ldots y_{9}, m_{1} \ldots m_{9}, \varphi_{0}, \varphi_{10}, Q_{0}$ and $Q_{10}$ (shown on the left of the resolving matrix in fig.5).

Fig. 7 and Fig. 8 show the diagram of deflections and the bending moment curve.

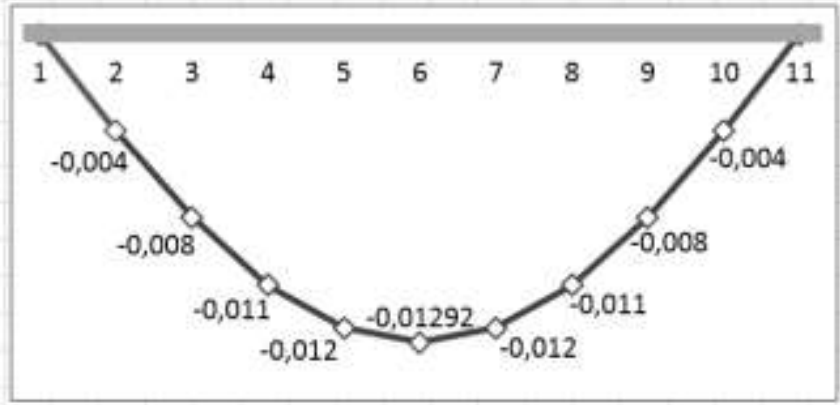

Fig.7. The diagram of deflections

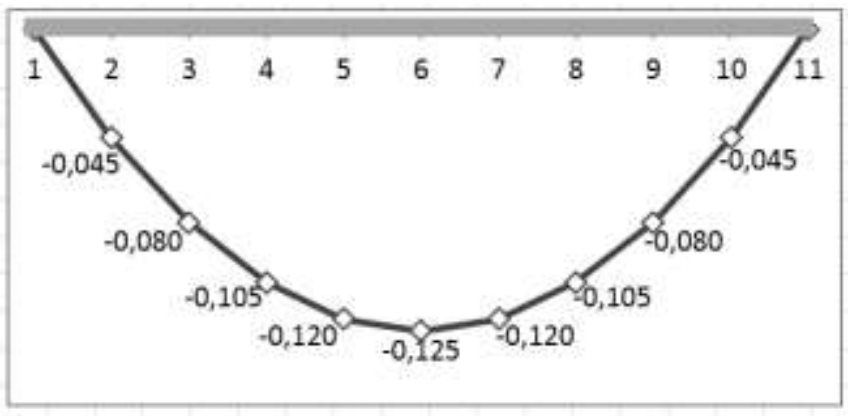

Fig.8. The bending moment curve

By analogy, the calculation apparatus is also formed for other boundary conditions. In Fig. 9 and 11 there are matrices for calculating unknowns for a beam with both pinched ends and for a cantilever beam, as well as moments for these beams (Fig. 10, 12).

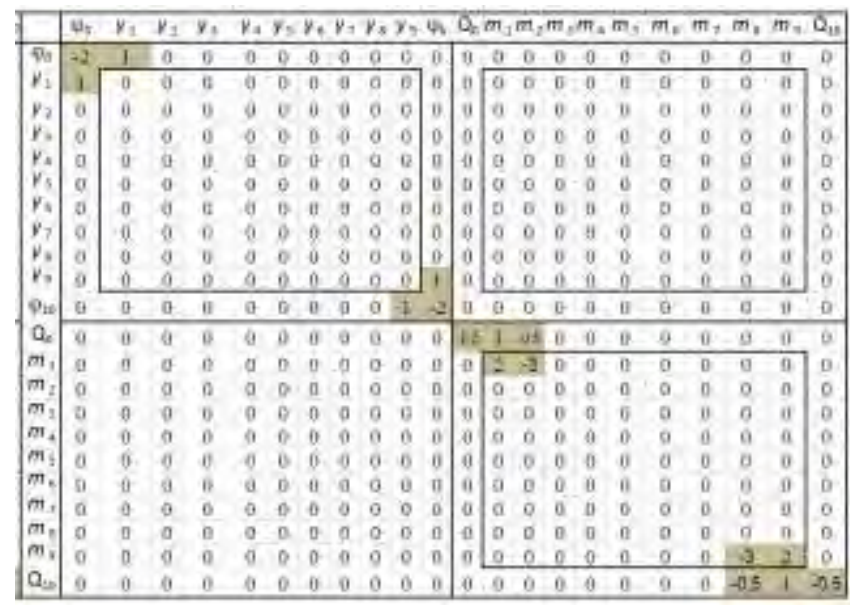

Fig. 9. The additional matrix for the beam with both pinched ends

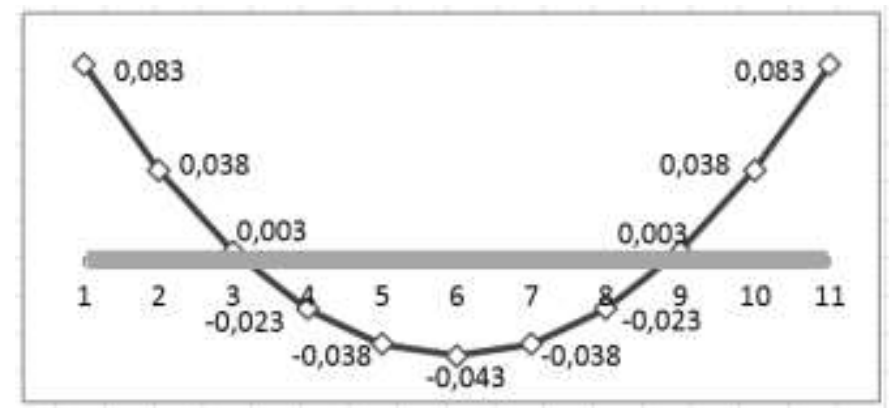

Fig.10. The bending moment curve for the beam with both pinched ends

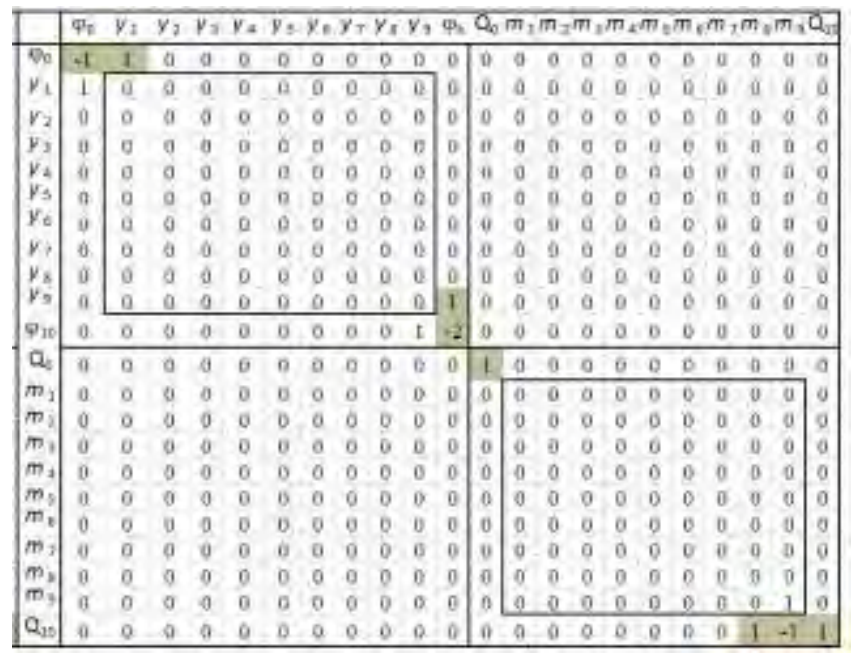

Fig.11. The additional matrix for a cantilever beam 


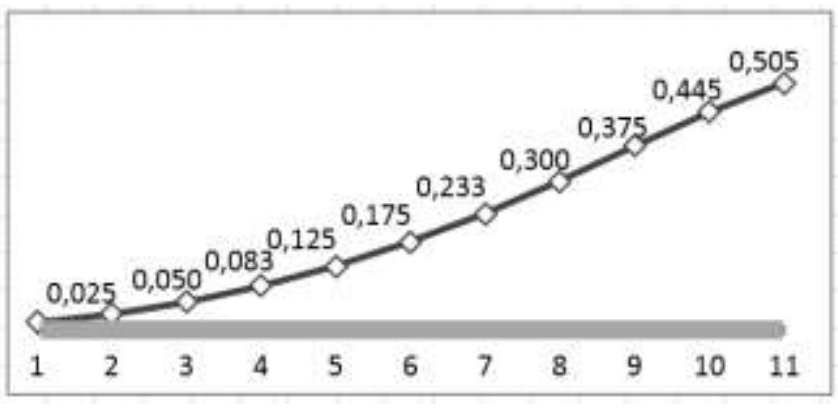

Fig.12. The diagram for a cantilever beam

\section{CONCLUSION}

Technology's versatility lies in the fact that for each variant of the initial data, a separate resolving matrix is created, that is, a result of a sum of two matrices. The first is the base matrix whose core is constant for all other matrices and is independent of the boundary conditions (the border columns and rows of this matrix are zero). And the second is an additional matrix, the core of which is filled with zeros, and the cells of the border columns and rows are filled with the values of the given boundary conditions. Examples of these matrices are given for different boundary conditions and the calculation results (diagrams of deflections and the bending moment curve).

Thus, the proposed approach to the creation of softwarealgorithmic computational complexes allows computer modeling of complex processes of behavior of a structure under various impacts on it.

Besides, the possibility of the user's independent modification of the data processing algorithm implemented in the considered method, taking into account the specificity of the particular problem being solved, makes it possible to simulate more accurately the conditions and parameters of the calculated structures, which significantly expands the practical application of the approach. Effective use of the entire arsenal of unique capabilities provided by modern table processors allows us to reach a new level of computerization of research activities.

\section{References}

[1] Yu.I. Uskov, S.Yu. Katerinina, M.A. Katerinina, "Matrix form of discrete analogue of generalized differential equation of curved axis of one-dimensional element", Vestnik VolgGASU, vol. 41(60), pp. 130138, 2015.

[2] Yu.I. Uskov, S.Yu. Katerinina, M.A. Katerinina, "Construction of a discrete analogue of the equation of bending of the rod on the basis of the modified method of spline approximation", Construction mechanics and construction engineering, vol. 2, pp. 39-43, 2015.

[3] V.A. Ignat'ev, Reduction calculation methods in statics and dynamics of lamilar systems, Saratov, Publishing house of Saratov University, p. 295, 1992.

[4] V.A. Ignat'ev, Thin-walled cellurar structures (methods for their analysis). A.A. Balkema/Rotterdam/Brookfield, p.621, 1999.

[5] G. Nürnberger, F. Zeilfelderb, "Developments in bivariate spline interpolation", Journal of Computational and Applied Mathematics, vol. 121(1-2), pp. 125-152, 2000.

[6] M.C. Lopez de Silanes, M.C. Parra, M. Pasadas, J.J. Torrens, "Spline approximation of discountinuous multivariate functions from scattered data", Journal of Computational and Applied Mathematics, vol. 131(12), pp. 281-298, 2001.

[7] E.J. Billo, Excel for Scientists and Engineers: Numerical Methods. A John Wiley \& Sons, Inc., p.477, 2007.

[8] N.G. Bandurin, V.A. Ignat'ev, "Method and Algorithm for the solution of essentially nonlinear ordinary integro-differential equations", Internet-Vestnik VolgGASU, vol. 2(7), 2008.

[9] D.E. Ivanov, T.V. Gromova, T.N. Shvetsova-Shilovskaya, O.V. Polekhina, "Conjugation technology development of the informationanalytical system for reliability indicators valuation of equipment at hazardous production facilities with foreign and domestic software products", Automation. Modern technologies, vol. 71(10), pp. 456-464, 2017.

[10] E.V. Akimova, E.D. Khokhlova, "Fast Fourier transform and its implementation in modern software", Fundamental and applied research in the modern world, vol. 18-1, pp. 134-137, 2017.

[11] N.A. Buzalo, S.A. Alekseev, Tsaritova N.G. "The engineering of nodes of spatial rod constructions of prefabricated buildings and structures" Construction and industrial safety, vol. 4(56), pp. 59-62, 2016.

T.L. Kobaliya, P.S. Chernov, A.S. Marutyan, "Light spatial and bar construction of covers", Sovremennaa nauka i innovacii, vol. 4(8), pp. 64-71, 2014. 